\title{
HYPERPYREXIA DURING GENERAL ANAESTHESIA: A CASE REPORT
}

\author{
Gérard Lavore, M.D. *
}

\section{Anaesthesia Complicated by Muscular Rigidity Following Induction, Terminating in Hyperthermia and Cardiovascular Failure}

The pattent was a healthy, muscular, 42-year-old white male presented for repair of a corneal wound of the eye. History revealed no previous illnesses. Physical examination was normal. The arterial blood pressure was 130/80, and the pulse was 76 per minute. The haemoglobin was $14.7 \mathrm{gm}$. Premedication was meperidine $50 \mathrm{mg}$, and atropine $0.6 \mathrm{mg}$, given at 9.00 P.M.

On arrival in the operating room the patient seemed in excellent health, if a little apprehensive. Anaesthesia was begun at 11.00 P.M. Induction was with 2.5 per cent thiopentone, $400 \mathrm{mg}$. and $60 \mathrm{mg}$. of succinylcholine with $2 \mathrm{mg}$. of decamethonium for intubation. Ventilation was done with 100 per cent oxygen. Immediately marked fasciculations appeared, followed by sustained contracture of the muscles of the jaw, neck, and limbs, making intubation impossible. A further dose of succinylcholine ( $60 \mathrm{mg}$.) and decamethonium ( $4 \mathrm{mg}$.) was given, to relax the contractions, and a further dose of thiopentone (100 mg.) was given to maintain anaesthesia. After ten minutes intubation was successful, and anaesthesia was continued with oxygen, nitrous oxide, and halothane, in a semi-closed circuit with absorption. During the period of difficulty, the patient was apnoeic, and pulmonary ventilation with mask was easily accomplished.

Throughout the operative period the blood pressure was maintained at $110 / 80$. The pulse, which was 100 per minute preoperatively, accelerated to 120 per minute after 45 minutes of anaesthesia, and stayed at this rate until the end of the operation, which lated 75 minutes. Respiration was controlled throughout the operation. At the end of the intervention the patient was not awake but was breathing spontaneously, if inadequately. He was kept intubated, and respiration was assisted. It was noted that the muscular contractures of the neck, abdomen, and limbs persisted.

Twenty minutes after the intervention the patient seemed to be still deeply asleep, colour was good, but the skin was hot, "almost burning." Carotid pulse was strong and regular at 76 per minute. It was noted now that the blood pressure was $70 \mathrm{~mm}$. $\mathrm{Hg}$ systolic on oscillation. Temperature was $104^{\circ} \mathrm{F}$. Methedrine (two successive doses) was given, and sodium bicarbonate, but there was no improvement. An aramine drip was installed, but still the blood pressure was not improved, and the pulse slowed and became irregular at 40 per minute. An E.C.G. was run, and showed what resembled an atrio-ventricular block. An Isuprel drip was installed. The heart accelerated somewhat, but the blood pressure remained low.

'Maisonneuve Hospital, Montreal 36, P.Q.

Can. Anaes. Soc. J., vol. 13, no. 5, Sept. 1960 
At 1.30 A.M. a neurological examination was done by a consultant. It revealed complete absence of reflexes. A lumbar puncture showed nothing abnormal. The temperature was now $107^{\circ} \mathrm{F}$. (rectal). It was then planned to cool the patient, but before hypothermia could be installed the vital signs rapidly deteriorated. External cardiac massage was performed without success. At 2.45 A.M. the patient was dead.

An autopsy showed pulmonary congestion and atelectasis, and was otherwise negative.

\section{RÉSUMÉ}

Il s'agit d'un homme blanc, bien musclé, en bonne santé, âgé de 42 ans qui s'est présenté pour faire réparer une blessure à la cornée. L'histoire et l'examen physique ne révèlent rien departiculier. Sa tension artérielle est de 130/80 et son pouls, de 76 par min. Son hémoglobine était de $14.7 \mathrm{gm}$. A 9 heures du soir, on lui donne en prémédication: mépéridine $50 \mathrm{mg}$. et atropine $0.6 \mathrm{mg}$.

A son arrivée d la salle d'opération, le malade semble en parfaite condition, mais un peu appréhensif. On commence l'anesthésie à 11 heures du soir avec une solution de $2 \frac{1}{2}$ pour cent de thiopentone: $400 \mathrm{mg}$, et, pour lintubation, de la succinylcholine $60 \mathrm{mg}$. et $2 \mathrm{mg}$. de décaméthonium. Nous avons ventilé le malade avec de l'oxygène pur. Immédiatement, nous avons observé des fasciculations, puis est apparue une contracture des masseters, des muscles du cou et des membres, ce qui a rendu impossible l'intubation. Dans le but de faire céder ces contractions, nous avons répété une injection de $60 \mathrm{mg}$. de succinylcholine et de $4 \mathrm{mg}$. de décaméthonium. Pour maintenir l'anesthésie, nous avons donné une autre dose de $100 \mathrm{mg}$. de thiopentone. Au bout de dix minutes, nous avons réussi l'intubation et nous avons continué l'anesthésie avec de l'oxygène, du protoxyde d'azote et de l'halothane en employant un circuit semi-fermé avec absorption. Au cours de nos difficultés, le malade était en apnée, mais il était facile à ventiler avec un masque. Au cours de l'opération, la tension artérielle s'est maintenue à 110/80. Avant l'opération, le pouls était de 100; après 45 minutes d'anesthésie il s'est accéléré à 120 et s'est maintenu à cette vitesse jusqu'à la fin de l'opération qui a duré soixante-quinze minutes. Nous avons controlé la respiration durant toute l'opération. A la fin de l'opération, le malade n'était pas réveillé, mais respirait spontanément, de façon presque normale. Nous lui avons laissé son tube et avons assisté sa respiration. Nous avons observé que la contracture musculaire de son cou, de son abdomen et de ses membres persistait.

Vingt minutes après l'opération, le malade semblait dormir encore profondément, sa coloration était bonne, mais sa peau était chaude, "presque brulante". Son pouls carotidien était fort et battait à 76 par min. A l'oscillation, sa tension artérielle systolique était à $70 \mathrm{~mm}$.Hg. Sa température était à $104^{\circ} \mathrm{F}$. On a donné de la méthédrine (deux doses successives) et du bicarbonate de sodium mais sans amélioration. Nous avons installé un goutte à goutte d'aramine, mais la tension artérielle est demeurée basse et le pouls s'est ralenti et est devenu irrégulier à $40 \mathrm{par} \mathrm{min}$. Un tracé électrocardiographique a montré ce qui ressemble à un blocage auriculo-ventriculaire. Nous avons installé un goute à 
goutte d'Isuprel: le pouls s'est accéléré un peu, mais la tension artérielle est demeurée basse.

A 1.30 hre du matin, un consultant fait un examen neurologique et observe une absence totale de réflexes. Le liquide céphalorachidien est normal. La température rectale est de $107^{\circ} \mathrm{F}$. Nous nous proposions à ce moment de refroidir la malade, mais avant que nous ayons eu le temps d'installer l'hypothermie, les signes vitaux se sont détériorés. Nous avons pratiqué le massage cardiaque sans succès. A 2.45 hres du matin, le malade mourait. A l'autopsie, on a constaté de la congestion pulmonaire, de l'atélectasie et, par ailleurs, rien de particulier. 